Service social

\title{
Réflexions à propos d'une expérience de formation des travailleurs sociaux sur le sida
}

\section{Catherine Tourette-Turgis}

Volume 38, numéro 1, 1989

Aspects psychosociaux du Sida

URI : https://id.erudit.org/iderudit/706422ar

DOI : https://doi.org/10.7202/706422ar

Aller au sommaire du numéro

Éditeur(s)

École de service social de l'Université Laval

ISSN

1708-1734 (numérique)

Découvrir la revue

Citer cet article

Tourette-Turgis, C. (1989). Réflexions à propos d'une expérience de formation des travailleurs sociaux sur le sida. Service social, 38(1), 37-43.

https://doi.org/10.7202/706422ar
Résumé de l'article

Le présent article relate une expérience de formation qui concerne 21 groupes de travailleurs sociaux répartis dans quatre régions de France. Après avoir mentionné comment s'est manifesté le besoin de formation, l'auteure décrit les contenus des programmes, l'approche pédagogique et les divers questionnements qui ont entouré cette expérience. 


\section{Réflexions à propos d'une expérience de formation des travailleurs sociaux sur le sida}

\section{Catherine Tourette-Turgis ${ }^{1}$}

Le présent article relate une expérience de formation qui concerne 21 groupes de travailleurs sociaux répartis dans quatre régions de France (Bretagne, Haute-Normandie, Île de France et la région AntillesGuyane). ${ }^{2}$ Ces stages ont lieu dans des instituts de formation de travailleurs sociaux conventionnés par la Direction de l'Action sociale et sont financés par la Direction générale de la Santé depuis 1987. Par ailleurs, des stages sont offerts spécifiquement aux personnels du Ministère de la Justice (Administration de l'Éducation Surveillée et Administration Pénitentiaire).

Cette expérience a déjà fait l'objet d'une présentation lors du $24^{\text {e }}$ Congrès international des Écoles de Service social en juillet 1988 à Vienne, de même qu'à la $5^{\mathrm{e}}$ Conférence internationale sur le SIDA, tenue à Montréal, en juin 1989. J'en reprendrai donc quelques éléments essentiels et ferai part des réflexions issues de cette expérience.

\section{La demande}

Dans le cadre de la prévention et de la lutte contre le sida en France, il a été estimé que les travailleurs sociaux avaient un rôle à jouer et qu'il était nécessaire d'envisager une formation appropriée. Il était important, dans la mise en place des programmes destinés aux travailleurs sociaux, d'éviter l'injonction à une formation concernant un sujet comme le sida.

Par contre, la Direction de l'Action sociale a suscité et impulsé des demandes de formation en respectant l'hétérogénéité et la diversité 
des régions, tout en posant le principe d'une approche pluridisciplinaire et multipartenariale.

\section{Les recommandations de formation}

Autant pour l'information que pour la formation, il est important, face à une maladie qui suscite tant de mécanismes de rejet, de peurs et d'incertitudes de la part des populations, que soient mis en place des espaces de paroles, de savoirs et de réassurance pour les travailleurs sociaux afin qu'ils puissent assurer la prévention et la gestion sociale de l'infection VIH.

C'est pourquoi la Direction de l'Action sociale a privilégié les formations longues ( 60 heures) fondées sur une approche pluridisciplinaire et multipartenariale ayant pour souci de confronter et d'initier les acteurs sociaux à tous les aspects de la maladie (médicaux, sociaux, psychologiques, juridiques et institutionnels), les préparant à comprendre non seulement la situation mais aussi l'évolution des réactions de la population et à s'y adapter.

Si la formation a pour but de favoriser une promotion individuelle et collective de chacun au rôle d'acteur dans une politique de prévention, les conditions en sont le partage d'un savoir dans un contexte soucieux de prendre en compte les systèmes culturels, les systèmes de valeurs et de normes des populations concernées au niveau épidémiologique par la maladie, tout en essayant de réduire les effets pervers de la sociologie implicite de l'épidémiologie pour ne pas trahir la vocation éthique de la Santé Publique.

\section{Les contenus des programmes}

Tous les programmes sont conduits dans une triple orientation: information, formation, recherche. En ce qui concerne les savoirs extérieurs, ils sont fondés principalement sur quatre approches : médicale, juridique, psychosociale, psychologique.

L'approche médicale doit jouer sur la légitimité et la vulgarisation d'une connaissance médicale. La réponse ne doit pas en être une d'autorité mais de connaissance, c'est-à-dire avoir lieu dans un contexte partagé. L'épidémiologie est d'un apport fondamental pour la prévention ; l'approche que nous proposons relève d'une épidémiologie d'intervention dans la mesure où il s'agit d'abord de mettre en évidence l'efficacité de mesures préventives au sein d'une population qui ne se croit pas forcément concernée, et ensuite de trouver des ajustements 
particuliers en fonction des populations auxquelles on s'adresse dans le cadre de la prévention.

L'objectif de l'approche juridique et institutionnelle est de permettre à des travailleurs sociaux d'introduire, dans la relation duelle instituée (assistant social-client, éducateur-jeune, infirmier-malade), la loi dans sa fonction réelle et symbolique.

Au-delà d'une approche juridique qui se limiterait à envisager la question du sida en regard de la législation propre à cette maladie, il s'agit de faire fonctionner la problématique du sida comme pouvant interroger la loi.

L'approche psychosociale se propose, par le biais de l'étude des comportements individuels et collectifs, de répondre à la question suivante : comment se situent et interagissent individus et groupes face à des situations de risques et d'incertitudes ? Toute stratégie d'intervention sociale voulant répondre à cette question qui est le pilier de la prévention, ne peut que prendre en compte la diversité des attitudes sociales et culturelles à l'égard de la sexualité, de la mort, de la maladie. Elle envisage l'individu dans une culture, un système de normes qui donne sens à ses actions quotidiennes et à ses interactions avec son environnement.

L'approche psychosociale permet de re-situer la sexualité dans une problématique plus large que celle qui consiste à l'aborder en termes de conduites, de comportements et d'attitudes. Au-delà des conduites sexuelles individuelles, c'est toute la structure des échanges dans une société qui est en jeu. Chaque société possède une structure économicosexuelle qui lui est propre, et développe un ensemble de normes adaptées à cette structure. Nous privilégions cette approche de la sexualité à une approche strictement psychologique ; si cette dernière est valable en soi à l'intérieur de son dispositif clinique, elle n'est pas utilisable en tant que telle dans le travail de soutien social exigé par le sida et dans le travail de prévention à mener au sein des communautés homosexuelle et hétérosexuelle. Trop proche dans ses effets d'un discours moralisateur, l'approche psychologique de la sexualité nécessite des compléments sociologiques, juridiques, anthropologiques, culturels et autres.

Bien qu'il soit justifié, du point de vue médical, de présenter le sida en privilégiant le réel médical de la mort, il faut y reconnaître le risque que, du point de vue psychologique, soit imposé à celui qui est réellement concerné par la maladie et à son entourage, le poids de nos fantasmes de mort. Alors que ce qui est essentiel pour un sujet séropositif ou malade, c'est de pouvoir reprendre pour lui l'image, portée voire supportée par l'extérieur, d'un être qui est un sujet vivant jusqu'à la dernière limite de sa vie. 
Tout travail de soutien psychologique doit faire en sorte que la société et les institutions concernées dépassent le stade de la "victimisation » de la séropositivité et de la maladie. Le sida n'est pas une maladie subie mais une maladie vécue, et il nous paraît important de mettre l'accent sur les façons de composer avec elle. La professionnalisation trop rapide de personnels dans des fonctions d'écoute et de soutien risque, si des précautions ne sont pas prises, de re-victimiser les patients et les malades. La victimisation du malade consisterait à travailler avec des savoirs construits comme des mesures d'urgence, à l'usage de personnes ou de populations dont on n'aurait pas eu le temps de recueillir les savoirs qu'elles-mêmes produisent et inventent chaque jour. C'est en ce sens qu'une psychologisation trop rapide de la séropositivité et du sida n'est pas sans coûts et risques pour les patients et les soignants. En effet, comment conduire des observations psychologiques ou enseigner une psychologie ou psychopathologie de la séropositivité ou du sida sans faire part, non seulement de la nature et des modalités de la demande du patient, mais aussi du vécu de cette demande par celui, bénévole, professionnel, ami, qui la reçoit?

\section{Problèmes épistémologiques}

Dans la mesure où, en France, nous ne disposions pas de tradition de formation sur le sida et que l'histoire de la syphillis et de la tuberculose avait été dénoncée comme l'histoire de pratiques non-éthiques, on peut dire que l'approche privilégiée en termes de prévention et de soutien a été le modèle anglosaxon (USA) importé en France par les premières associations de défense et de soutien de malades (AIDES). En termes de méthodes, cette approche se situe dans le courant des thérapies comportementales (visualisation positive, psychonutrition, safer-sex...) en rupture avec une autre tradition de formation socioclinique française des travailleurs sociaux fondée sur la psychanalyse.

L'utilisation d'un modèle exogène pour le soutien psychosocial n'a pas fini de nous interroger sur nos difficultés à nous approprier le sens et la signification de cette maladie dans notre histoire et notre culture. Ces réflexions nous ont amené à insister, dans les groupes, sur la constitution de l'histoire du sida et à repérer ses modes d'insertion dans chaque société où la maladie survient. Cela permet de dégager les arbitraires culturels sous-tendus dans la production et la multiplication des catégories discriminantes que le discours sur le sida engendre. L'histoire de la propagation du virus dans le monde nous permet aussi de démêler les hypothèses irréfutables des assertions révélant un racisme 
scientifique international (effets pervers des débats sur l'origine du virus).

\section{Le groupe BALINT}

L'intensité des réactions dans les groupes a suscité chez les formateurs la demande d'un groupe BALINT ${ }^{3}$. Un des premiers effets du groupe BALINT a été de réduire la portée "apostolique " des stages due à nos implications dans des associations de soutien et de défense des malades. Un autre effet immédiat a été d'atténuer la primauté de la rationalité dans les processus de connaissance au profit de la prise en compte des dimensions fantasmatiques concernant l'infection VIH, ce qui, conséquemment, a remis en question l'organisation des contenus des sessions, notamment en ce qui concerne l'approche médicale.

Dans la mesure où, dans la société, la médecine ne peut répondre au sida (comme à toute grande maladie transmissible) qu'en termes de stratégie d'attaque, de lutte, d'extermination, d'éradication, le sens de la maladie est implicitement véhiculé par un ensemble de métaphores guerrières et particulièrement agressives. Cela n'est pas sans effet sur la perception qu'en ont les acteurs sociaux qui, se trouvant ainsi en prise directe et imprévue avec son réel, s'en défendent par une série de mises à distance. Ces défenses prennent tantôt la forme de la peur, de l'angoisse, de la sidération, du désespoir, et provoquent des mouvements d'identification et de désidentification aux victimes de la maladie et personnes exposées aux "risques" indiqués par les classifications épidémiologiques.

Aussi, avons-nous l'intention, dès nos prochaines sessions, de privilégier un travail clinique de groupe en préalable à toute approche médicale, pour réduire le poids des croyances et des fantasmes constituant à eux seuls des facteurs puissants de résistance et d'inhibition intellectuelle (ex. : fantasmes archaïques sur le sang...)

\section{Les institutions face au sida}

Parallèlement à la formation, nous conduisons deux recherches sur les aspects institutionnels concernant l'accueil des personnes touchées par le virus VIH, dont l'une porte sur la prise en charge des personnes toxicomanes séropositives au $\mathrm{VIH}$.

L'accueil des individus séropositifs semble nécessiter pour les institutions non seulement une reconversion fonctionnelle mais la mise en place d'une prévention des pathologies latrogènes institutionnelles. 
On a bien vu comment la simple réintroduction des règles d'hygiène (comme l'utilisation des gants dans les soins) non seulement modifiait les niveaux de sociabilité entre soignants et patients, mais appauvrissait le sens commun des opérations signifiantes de la vie quotidienne en milieu institutionnel (vaisselle, linge, plateau de nourriture, chambre) pour en faire des mesures d'exception.

\section{Les effets pervers}

L'insistance sur la contextualisation des savoirs et des informations ne protège personne de leur normalisation et de leur usage pervers. Tout technicien du savoir ou de la formation doit être à même de repérer les mésusages de la connaissance et éviter les dérapages intellectuels menant à une position anti-éthique.

Il va de soi que l'ignorance ou la mauvaise utilisation des connaissances sont à interpréter en termes de mécanismes de défense contre l'angoisse et l'incertitude. À ce titre, nous devons aussi être à même de travailler avec les lapsus et les actes manqués de la connaissance et des pratiques. Sur ce sujet plus que tout autre, les processus de formation requièrent la mise en place de procédures devant contenir l'ensemble de ces incertitudes, en acceptant d'apprendre des personnels en formation ce qu'ils ont à dire.

\section{Perspectives}

Récemment, nous avons pris conscience de notre manque de connaissances, en France, sur l'aspect psychosocial féminin de l'infection $\mathrm{VIH}$. Son incidence croissante chez les femmes pose des problèmes d'attitudes à adopter, notamment face à la grossesse. Les travailleurs sociaux ont d'ailleurs fait une demande de formation en ce sens. De même, nous avons ouvert un autre thème concernant la prise en charge et le soutien psychosocial à apporter à la seconde génération des personnes touchées par le VIH: les enfants.

Bien sûr, il faut agir et réagir au même rythme que la maladie. Mais il faut surtout penser, vivre et évoluer au rythme des réactions des populations. Ce sont les populations qui nous apprennent quotidiennement ce qu'est le sida et, en ce sens, la formation consiste à reprendre ce savoir dans un espace approprié de façon à mettre en œuvre des pratiques d'intervention sociale d'intérêt collectif où les populations seraient actrices de leur santé. 


\section{Notes}

1 En collaboration avec Jacqueline Bonneau, conseillère technique à la Direction de l'Action sociale, Ministère de la Solidarité, de la Santé et de la Protection sociale.

2 D'autres stages sont mis en œuvre dans ces mêmes perspectives dans d'autres régions de France (Auvergne, Provence, Alpes, Côte d'Azur, Rhône Alpes).

3 Du nom d'un psychiatre britannique qui a conçu des ateliers s'adressant aux médecins et visant spécifiquement leur formation en relation d'aide. 